\title{
EI ROL FEMENINO EN EL MERCADO LABORAL Y EL DESEMPLEO EN COLOMBIA
}

\author{
Kathleen Cataño Cadavid \\ Escuela de Negocios, Gestión y Sostenibilidad \\ Programa de Economía \\ kathleen.cadavid@gmail.com \\ Natalia Diaz Gonzalez \\ Escuela de Negocios, Gestión y Sostenibilidad \\ Programa de Negocios Internacionales \\ natadg23@gmail.com \\ Julieth Rodriguez Torres \\ Escuela de Negocios, Gestión y Sostenibilidad \\ Programa de Negocios Internacionales \\ juliethroto95@gmail.com
}

\section{Resumen}

En el presente artículo trabajamos respecto al desempleo que presentan las mujeres en Colombia, y en cuáles ciudades es más evidente este fenómeno, gracias a una serie de aspectos que hoy en día se siguen manteniendo. Se realiza una investigación de tipo cualitativo con el fin conocer cuál es la realidad que afrontan las mujeres en el mercado laboral colombiano.

Además, se evidencia la falta de oportunidades laborales debido al hecho de ser mujer y la desigualdad que hay por ello, ya que terminan convirtiéndose en un costo a largo plazo para las empresas, en dado caso de quedar en embarazo. Sin olvidar que el estado civil también se convierte en un factor determinante a la hora de la contratación del personal femenino dentro de las organizaciones y la diferencia salarial que se mantiene por solo ser mujer.

Palabras Claves: Desempleo; mujeres; Colombia; mercado laboral; desigualdad. 


\section{Introducción}

A través de este artículo, se pretende evidenciar las condiciones en que se ha desarrollado la participación de la mujer colombiana en el mercado laboral y la incidencia que ha tenido esta situación en el índice de desempleo del país. A partir de la clase de Teoría de las Organizaciones y la relevancia que tiene en la actualidad trabajar por una sociedad con igualdad de condiciones, surge el interés por conocer la realidad del rol femenino en el campo laboral colombiano. Se toma como base la teoría del comportamiento, la cual hace énfasis en el ser humano como persona dentro de la organización; se deja de ver al empleado como una máquina, y toma protagonismo el desarrollo personal.

Actualmente se habla de la participación de los sujetos en el mercado laboral, pero no de la desigualdad de oportunidades que este les ofrece. La población femenina está tan estigmatizada en tener un rol de cabeza de hogar y ama de casa, que aunque es muy válido, hace que la participación de ellas en el ámbito laboral sea muy complejo, y más si se le suman factores como educación y estrato socioeconómico.

Aunque en la mayoría de las vacantes, las empresas promueven una participación igualitaria de los individuos, los perfiles requeridos en su mayoría describen cualidades que son atribuidas al género masculino; tales como la independencia, la seguridad, el liderazgo, entre otras. Características que fácilmente una mujer puede obtener, estas alimentan los estereotipos que terminan convirtiéndose en una desventaja para las ellas mismas al momento de intentar acceder a ofertas laborales.

Algunas cifras evidencian que la cantidad de oferta laboral no es un medidor de igualdad de oportunidades, como lo dice la OIT (2018) "la tasa mundial de participación femenina en el mercado laboral fue del 48,5\%, 26,5 puntos porcentuales más bajan que la de los hombres", es decir, el funcionamiento del mercado ha convertido a las mujeres en una población vulnerable. Por una parte, aunque logren acceder a los empleos, no son consideradas como iguales dentro de las organizaciones, esto se ve reflejado en la amplia brecha salarial que se ve hoy en día. 
Se evidencia también que en la mayoría de los casos, aunque tengan el mismo nivel educativo y cargos similares o iguales a un hombre, su remuneración es menor.

Aunque si bien, según el DNE (2019), Colombia es uno de los países de Latinoamérica que cuenta con mayor participación de mujeres en el mercado laboral, en puestos de alto nivel, no se ve reflejado en los datos del DANE que muestran una tasa de desempleo en mujeres del $16,9 \%$, superior al de los hombres en un 7,1\%. Gracias a esto se ven obligadas a emplearse en trabajos informales, que no les ofrecen las garantías mínimas exigidas por el Estado. Según cifras del DANE, del total de empleados que perdieron su trabajo el año pasado, la población que se vio más afectada fueron las mujeres con un $85 \%$ de personas, esto se transcriben a 478 mil mujeres.

Como se observará en este trabajo, la falta de oportunidades hacia la población femenina en el mercado laboral tendrá una gran incidencia en el desempleo, ya que al no estar haciendo parte de las actividades que la economía considera productivas, es mano de obra que no se está aportando al desarrollo y crecimiento del país.

\section{Antecedentes}

Los diferentes cambios económicos y culturales siempre han sido un problema para el crecimiento de la oferta laboral dentro del país, gracias a que la evolución que aún se tiene no va de la mano con lo existente, siempre hay un retraso. Esto influye en un índice mayor de empleo en el personal masculino puesto que, se consideraba a la mujer un "sexo débil" y de poca adaptabilidad frente algunas labores que solo se asocian al género masculino.

Otros factores que influyen en la contratación del personal tanto masculino como femenino son la aparición de algunos trabajos que solo están relacionados con diferentes épocas del año, lo que genera una tasa de desempleo más alta en ciertas temporadas y en ciertos sectores respectivamente. Como, por ejemplo, los recolectores de café, los vendedores o auxiliares de un producto en época vacacional, y los trabajadores en empresas de flores y follajes de tipo exportación.

La entrada de tecnología no podía quedar atrás, puesto que con ello se aumenta la productividad de la empresa y se disminuye el ingreso de la participación de las personas dentro de la misma; sin olvidar que se capacita mucho más el personal masculino para este 
tipo de actividades que aparentemente necesitan de la intervención de un hombre. De igual forma, las horas máquina son mucho más económicas en contraste de la hora de un hombre laborada, ya que no requieren de una serie de gastos adicionales como lo son las vacaciones, seguridad social, días de descanso, pagos de utilidades (en caso de que la empresa las genere), primas y licencias (tales como la de adopción, maternidad y lactancia), estas últimas afectan directamente a las mujeres.

Por otro lado, hay trabajos que por su poca demanda fueron desapareciendo del mercado, debido a que el consumo disminuyó e hizo que estas empresas que brindaban fuentes de empleo recortan su personal para poder mantenerse en pie o en el peor de los casos cerrar la misma. También hay otras variables a la hora de un recorte de personal dentro de una empresa, como el bajo rendimiento en cada una de las labores que se debe desempeñar como trabajador y su representación monetaria, sin contar las consultorías externas que hacen una evaluación detallada del rendimiento de la productividad versus la ganancia que se puede generar.

Otro desafortunado factor presentado en el país es el del desplazamiento forzado, el cual lo sufren algunas personas debido a diferentes conflictos territoriales en el país, esto genera movilizaciones a las grandes ciudades como Bogotá, que recibió entre enero y noviembre del 2018 un aproximado de 30,517 personas, lo que incrementa el número de trabajos informales por dicha acción.

Los desastres naturales también logran afectar ciertos sectores de nuestra economía, hacen el campo laboral lento gracias a la reinversión que se debe hacer en cada uno de los sectores afectados por dicho evento, como la ola invernal que se presentó en el país en el año 2011, que dejó daños en infraestructura en algunas regiones y que necesitó de 81 mil millones de pesos para hacerle frente.

Ahora bien, cada una de estas causas de desempleo mencionadas anteriormente, influyen de una manera negativa en la contratación del personal femenino, puesto que el solo hecho de ser mujer las pone en desventaja, por el costo que representa la licencia de maternidad y cada uno de los agregados que este proceso natural conlleva. En la actualidad esto ha cambiado aún más porque dicha licencia se 
ha extendido, lo que significa un incremento en costos y la contratación de más personal mientras caduca dicha licencia.

Por último, la poca demanda laboral en actividades que requieren fuerza y que son asociadas netamente al género masculino, junto con el pensamiento retrograda que se mantiene de que las mujeres genéticamente están habilitadas para ciertas actividades, hace que se conviertan en un blanco fácil a la hora de buscar empleo y con esto una opción más sencilla de desechar dentro del mundo laboral.

\section{Metodología}

Se realizó una investigación de tipo cualitativo, con el fin de evidenciar la incidencia que conlleva la desigualdad de participación en el mercado laboral de las mujeres. Según Taylor y Bogdan (1987), la metodología cualitativa es un modo de hacer frente al mundo empírico estudiando el contexto de las personas y las situaciones actuales en las que se encuentran. Esto da como resultado una investigación que produce datos descriptivos que según Arias (2006), permiten medir cada una de las variables que representan los objetivos de la investigación y el alcance de esta.

Por lo cual, se elaboró una encuesta dirigida a administrativos y personal de recursos humanos, para conocer la importancia que tiene la participación de la mujer dentro de las organizaciones y las consecuencias que esto implica.

La población encuestada fueron 100 mujeres, en un rango de edad entre 18 y 29 años, pertenecientes a estratos sociales 1,2 y 3 ; donde se pudo evidenciar que la importancia que tiene el género en la contratación del personal se justifica con el diseño de los perfiles laborales, pero al final se termina reflejando un desequilibrio que responde a la concepción de estereotipos relacionados con la maternidad. Ya que, aunque no se exprese abiertamente, para las organizaciones representa un costo mayor tener que enfrentarse a un posible periodo de gestación.

Por este motivo se considera que las mujeres son menos productivas en el ámbito laboral. Se logró determinar que en el rango de edad mencionado la población femenina se 
encuentra en una condición de vulnerabilidad, debido a que es en este rango de edad donde se presentan mayores casos de embarazo.

Además, encontramos que el estrato en el que se encuentran las mujeres tiene una relación directa con su nivel de vulnerabilidad, esto gracias a que al contar con una educación básica se perpetúan las relaciones de dependencia y una consecuencia de esto se ve reflejada en el tipo de empleo al que acceden, usualmente son empleos de tipo informal que no permiten que tengan continuidad en el mercado laboral.

\section{Marco teórico}

El mercado laboral tiene una connotación importante en la sociedad ya que está ligado con el crecimiento económico de los países, al ser ese el espacio donde se mide la productividad de los individuos. Lo estructuran las organizaciones y las oportunidades de empleo que generan, las cuales están sujetas al lugar y la época del año, factores que tiene una gran incidencia en la productividad. Se define así el mercado laboral como la reunión de los empleos. (Chiavenato, 2000).

De acuerdo al lugar, el mercado tiene unas características y tendencias particulares. En Colombia, al medir el desempeño del mismo, las opiniones de la efectividad difieren de acuerdo a los sectores. Por un parte, desde las instituciones que lo estructuran, se considera como un espacio dinámico, adaptativo, que logra responder de acuerdo a sus capacidades a la generación de empleo y a unas condiciones salariales óptimas. Sin embargo, desde la perspectiva de aquellos que buscan ingresar al mismo, se considera como un espacio rígido, que no ofrece oportunidades y que se inclina a defender únicamente los derechos de las organizaciones (Portafolio, 2013).

La perspectiva de los colombianos frente a la respuesta del mercado de acuerdo a la demanda de ofertas laborales en el país es negativa, dicha inconformidad se ve reflejada en la encuesta realizada por Adecco (2017) en 1a que los colombianos expresaron cuáles consideran sus principales obstáculos al momento de conseguir empleo, el 22\% afirmó que una de las barreras más frecuentes es la falta de experiencia, y el $21 \%$ relaciono la falta de empleo con la edad (TIEMPO,2017). La incertidumbre que genera el mercado laboral colombiano tiene una connotación 
importante en la economía del país, ya que las organizaciones diseñan perfiles bajo unos estándares que no coinciden con la formación de la mayoría de la población que se encuentra en búsqueda de empleo. No se trata de modificar los estándares de calidad en las organizaciones, sino de trabajar porque los colombianos tengan una formación educativa de alta calidad, que permita abrir el campo de sus oportunidades laborales.

$\mathrm{Al}$ analizar los sectores que han sido altamente vulnerables al momento de conseguir empleo, la lista es encabezada por los jóvenes, los adultos mayores, la población con un nivel educativo bajo y las mujeres, las cuales, han librado una fuerte lucha en busca de igualdad de oportunidades en este campo. Según Dinero (2019), el bajo rendimiento del mercado laboral se debe a la desaceleración de la economía, la cual en el último año eliminó cerca de 775.000 puestos de trabajo. Estas acciones perjudican directamente a la población, siendo los más afectados los jóvenes y las mujeres, que cuentan con una tasa de desempleo de 18,5\% y 13\%, respectivamente.

Según el Instituto Nacional Sindical (ENS) se continúa reportando en el país que las actividades económicas que realizan con mayor frecuencia las mujeres son las que se les han aludido históricamente, que tienen una clara tendencia a empleos informales. Se continua con la concepción de que la principal labor del género femenino es el cuidado del hogar (TIEMPO, 2019).

La brecha de género en Colombia es evidente y tiene una gran influencia en el crecimiento económico del país. Saber que, en un mercado concebido para competir por oportunidades siempre se llega en desventaja, gracias a que la competencia no obedece a una muestra de capacidades, donde se pone a prueba el conocimiento y las habilidades, sino a un espacio donde se evidencia una arraigada ideología de género, que condiciona las actividades económicas en las que se puede participar, no es tarea fácil, como afirma Alicia Arango, Ministra de Trabajo (2019), "La mujer colombiana es motor de desarrollo económico y tenemos que aprovechar todos sus virtudes para sacar adelante el país adelante, y para que puedan acceder al trabajo y al emprendimiento".

De acuerdo con esta situación, es pertinente analizar qué sucede con esta población que no logra emplearse. Aunque el mercado este concebido para reunir la fuerza laboral, se evidencia que la oferta no es proporcional al número de demandantes, por lo cual no se logra ocupar toda la mano de obra disponible. 
En Colombia, el desempleo muestra una clara tendencia de acuerdo a la ideología de género. Se observa que ser mujer en el mercado laboral tiene una gran desventaja, "La tasa de ocupación de los hombres es de 67,6\%, mientras que para las mujeres es de 46\%, una diferencia de 21,5 puntos porcentuales" (Dinero, 2019).

Gráfica 1: Brecha en la Tasa de Desempleo entre ciudades

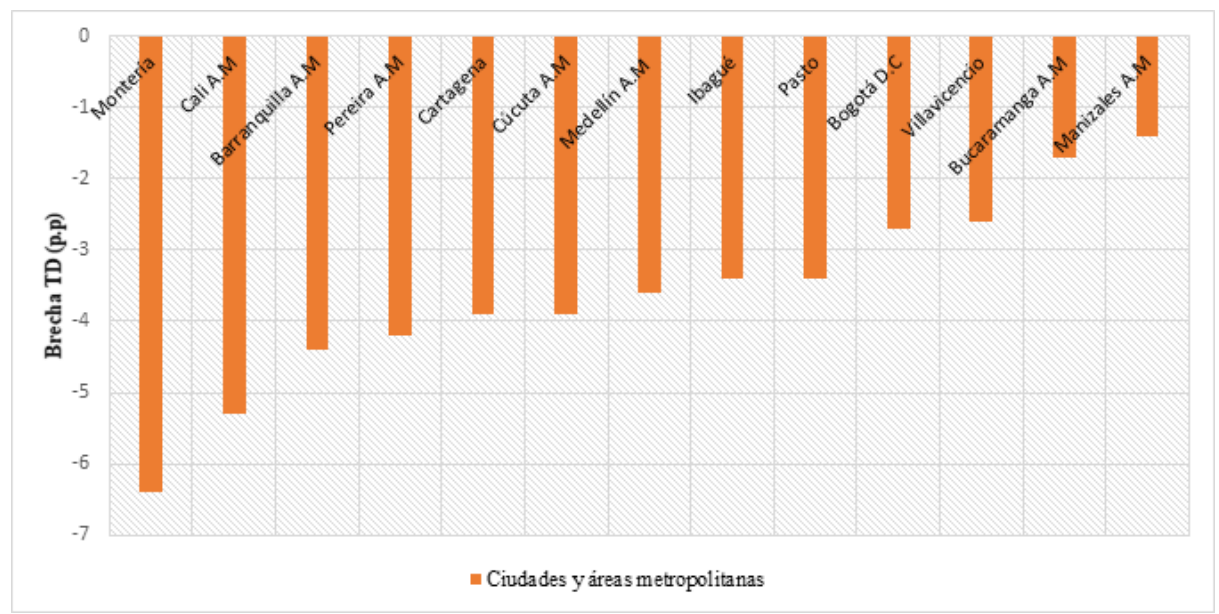

Fuente: DANE (2019).

Las cifras evidencian un mercado con ofertas dirigidas a aumentar el nivel de desigualdad, con fuertes restricciones que han incrementado el desplazamiento de la mano de obra femenina a actividades que la económica no considera productivas, y como consecuencia se observa una participación cada vez menor. Esta situación ha sido invisibilizada a través de indicadores que muestran crecimiento económico, pero no el coste real del mismo. Por ello, es necesario plantear un análisis que permita identificar el verdadero impacto que ha generado esta problemática en la sociedad y principalmente en el país.

\section{Recolección de datos:}

En Colombia, las mujeres ocupan el 50,7\% de la población, lo que se traduce a 24,5 millones de individuos. Aunque si bien las mujeres cuentan con la mayor fracción de individuos dentro del país, igualmente tienen la mayor tasa de desempleo e informalidad dentro del mismo. Más de 9 millones de mujeres se encuentran inactivas, y del total de mujeres que están laborando u ocupadas, $73 \%$ ocupan sectores tales como hostelería, comercio, servicios, y en restaurantes. 
Según cifras del DANE, la tasa de desempleo del año pasado a el año presente ha aumentado en un 1,6\%, lo que quiere decir que la tasa actual para el mes de agosto de 2019 fue $10,8 \%$.

Gráfica 2: Tasa de desempleo (2018-2019)

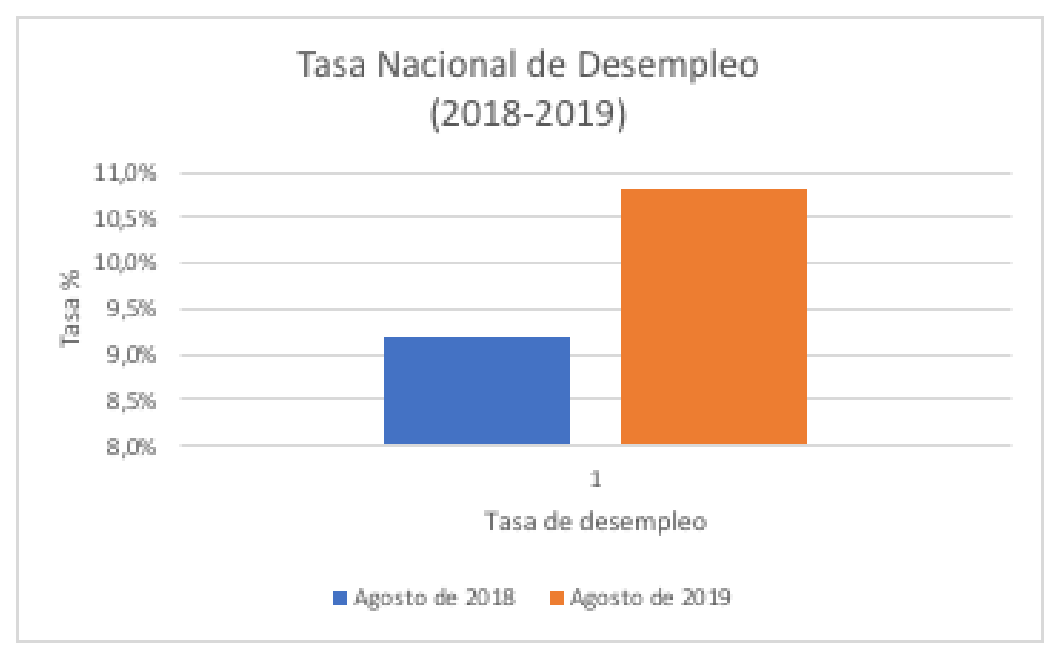

Fuente: DANE (2019).

Esta variación en el desempleo sostiene consecuencias importantes en la economía colombiana; ya que meses atrás ha aumentado la tasa de desocupados, lo que afecta de forma directa la tasa de desempleo. También las empresas están minimizando las vacantes de trabajo, como se menciona anteriormente.

Análisis de los datos:

Gráfica 3: Tasa de desempleo en mujeres por edad (2018-2019)

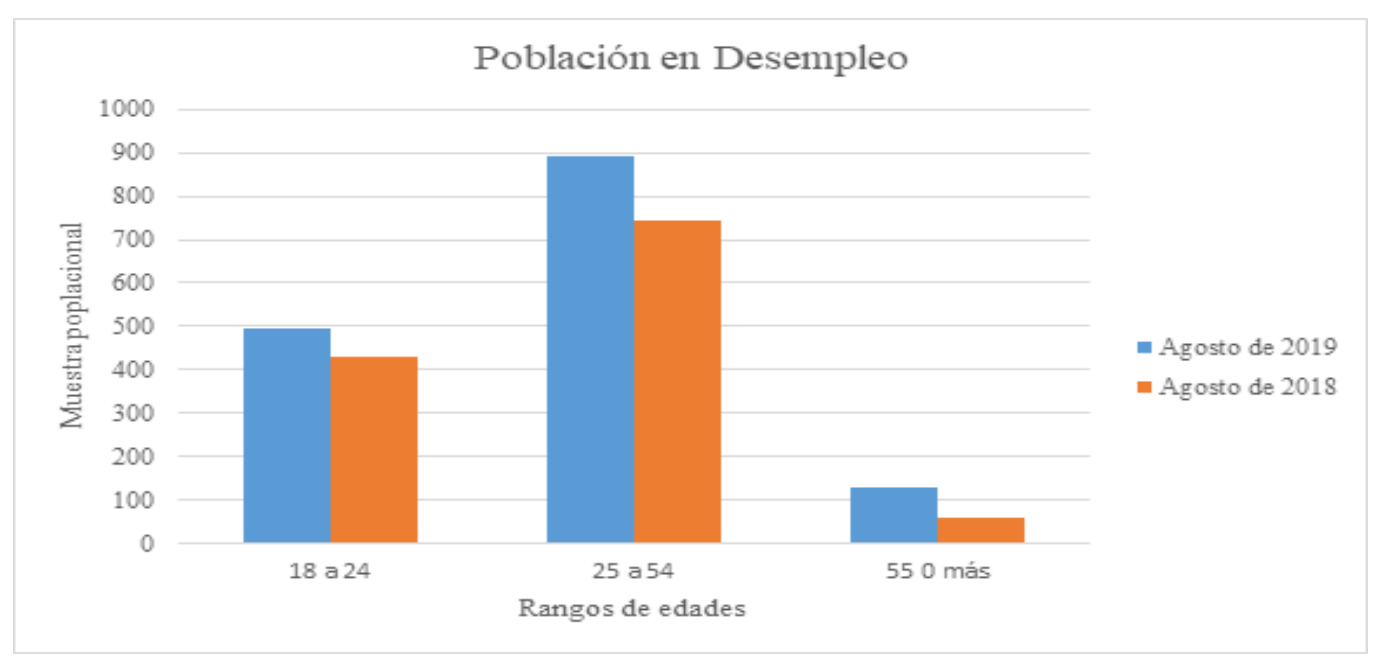

Fuente: DANE (2019). 
Las mujeres y los jóvenes como principales afectados en el tema, cuentan con una tasa de $13 \%$ y $18,5 \%$, respectivamente. Las mujeres se encuentran en un $4,6 \%$ de desempleo más alto que el de la población masculina desempleada; por consiguiente, la población más afectada por el desempleo en los últimos meses ha sido la juvenil femenina. Como manifiesta la congresista vallecaucana Catalina Ortiz en su cuenta de Twitter, "Ser joven y mujer es un doble obstáculo para entrar al mercado laboral. Entre abril y junio de 2019 el desempleo joven para hombres fue de 13,8\% mientras que el de las mujeres fue de $21,7 \%$ “.

Totalmente de acuerdo con la congresista, hacemos énfasis en que esta diferencia considerable hace pensar que hoy en día sigue habiendo un problema en cómo se organiza el mercado laboral en el país. Esto se puede evidenciar en ciudades como Montería, Barranquilla y Cartagena, donde la brecha de desempleo entre géneros es amplia.

Por otro lado, la brecha salarial es un tema que va de la mano con la diferencia de género, y visto desde el mercado laboral tiene consecuencias en el desempleo. Como se ha mencionado anteriormente, para el año 2019 ha aumentado la tasa de desempleo, y esto conlleva consecuencias para los trabajadores temporales, los menos calificados y las mujeres.

Tabla 1: Distribución de mujeres y hombres ocupados por rama de actividad (miles) Total nacional

$\begin{array}{rrrrrr}\text { EMPL } & \text { TRABAJADO } & \text { PATRÓ } & \text { EMP } & & \text { C } \\ \text { EADO DOMÉSTICO } & \text { R SIN REMUNERACIÓN } & \text { NO EMPLEADOR } & \text { LEADO DEL } & \text { UENTA } & \text { EMPL } \\ & & & \text { GOBIERNO } & \text { PROPIA } & \text { PARTICULAR }\end{array}$

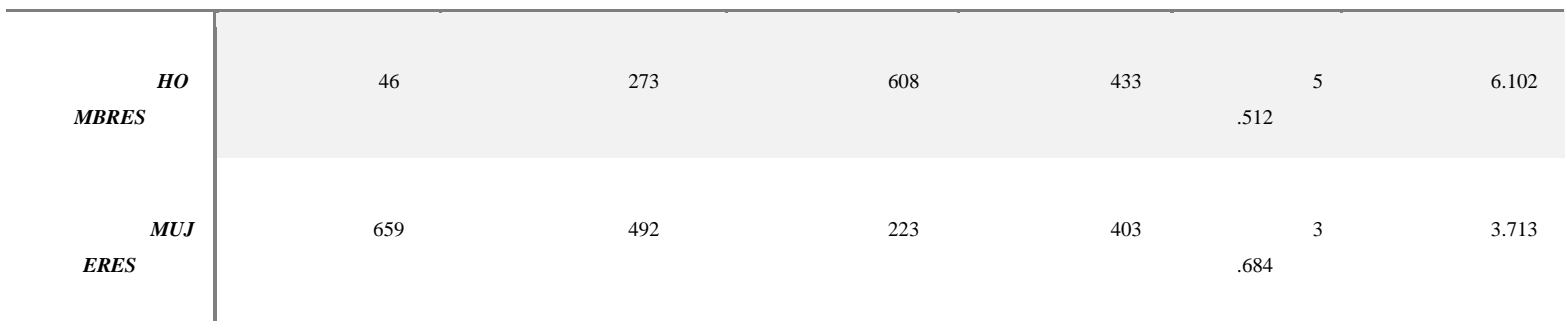

Fuente: DANE (2019).

Según la ONG, el 78\% de ofertas laborales van dirigidas a hombres, pero en su gran mayoría las mujeres son las que aplican a las mismas. Esto se debe al 
pensamiento que se tiene de que la mujer busca tener familia y mueren por la maternidad, afirmación que no en todos los casos es cierta o deseada.

Para la organización contratar una mujer trae a futuro muchos gastos y trámites que se ahorrarían a si contrataran un hombre. Gastos tales como, salario para la mujer mientras está en su tiempo de licencia, como para la persona que suplirá sus funciones mientras esta está acabando su licencia; como disminuciones en la productividad de las gestantes; y claramente falta de confianza en las capacidades de las mujeres. Según revista Dinero, la brecha salarial entre hombres y mujeres actualmente supera el $17 \%$.

\section{Conclusiones}

El comportamiento del mercado laboral actual no garantiza la igualdad del cumplimiento de los derechos entre hombres y mujeres, es decir no se aplican con las mismas condiciones. Por esta razón, es necesario que los gobiernos a nivel mundial legislen a favor de la igualdad de género para que la oferta laboral no funcione a partir de premisas discriminatorias, sino que garantice a hombres y mujeres una competencia a partir de sus capacidades profesionales.

El mercado laboral se ha estructurado bajo arraigadas ideologías de género, que de manera silenciosa han hecho que el papel de la mujer sea relegado, esto se evidencia en la falta de oportunidades que el sector les ofrece. En la mayoría de los casos, los perfiles se diseñan a partir de la búsqueda de competencias que son atribuidas al género masculino. Las empresas han sido el instrumento ideal para reproducir estos estereotipos, y por este motivo la oferta laboral siempre ha estado dirigida hacia el género masculino.

La diferencia salarial es un factor inequitativo entre los géneros, puesto que las competencias de cada ser humano (hombre vs mujer), no son pieza clave a la hora de recibir la remuneración económica pertinente. Por el contrario, es sabido que la brecha entre salarios es mucho más grande entre ambos, aunque se desenvuelven en el mismo campo laboral y con las mismas obligaciones.

A pesar del crecimiento que tiene el campo laboral colombiano y mundial, y con ello las reformas socioculturales que se presentan en el día a día, se evidencia la conservación de las diferentes maneras de discriminación laboral por las que son sometidas las mujeres, las cuales son de difícil eliminación dentro del campo laboral mismo. 
El tener un mayor nivel educativo, abre la posibilidad de conseguir un mejor desempeño en el campo laboral y con esto una mayor retribución económica, convirtiendo a la mujer en una ficha clave dentro de la organización económica existente en el país, sin embargo, no garantiza una igualdad en las ofertas laborales existentes.

Las mujeres se ubican en ciertos sectores laborales porque son víctimas de la discriminación ocupacional, lo que las lleva a desenvolverse en cierto tipo de actividades, con ciertos roles y reduce las posibilidades de emplearse con respecto al rol masculino, generando una serie de obstáculos y disminuyendo la probabilidad de entrar al campo laboral y aumentando así la tasa de desempleo. 


\section{Bibliografía}

Dane, (2019). Recuperado de: https://www.dane.gov.co/index.php/estadisticas-portema/mercado-laboral/empleo-y-desempleo

Oficina Internacional del Trabajo. (2018). Perspectivas sociales y del empleo en el mundo: Avance global sobre las tendencias del empleo femenino 2018. Recuperado de https://www.ilo.org/wcmsp5/groups/public/---dgreports/---dcomm/--publ/documents/publication/wcms_619603.pdf

Revista Dinero, (2019). ¿Qué es lo que está pasando con el mercado laboral?. Recuperado de https://www.dinero.com/edicion-impresa/coyuntura/articulo/cuales-son$\underline{\text { las-cifras-de-desempleo-en-colombia-a-agosto-de-2019/277391 }}$

Revista dinero, (2019). Ser mujer sigue siendo una desventaja en el mercado laboral colombiano. Recuperado de: https://www.dinero.com/pais/articulo/cuales-son-las-cifras$\underline{\text { de-desempleo-de-las-mujeres-en-colombia/275660 }}$

Chiavenato, Idalberto. (2000) Administración de recursos humanos. Recuperado de: https://www.google.com/url?sa=t\&source=web\&rct=j\&url=https://cucjonline.com/bibliot eca/files/original/f37a438c7c5cd9b3e4cd837c3168cbc6.pdf\&ved=2ahUKEwiQ b7d9Pbl AhVG11kKHYZvDjAQFjABegQIARAB\&usg=AOvVaw1NZP9Z_ysF7tQ09QoXfmiL

Carrasquilla, Contreras, González, Guzmán, y Pérez. (2010). Fecundidad y participación de la mujer en el mercado laboral en la Costa Caribe y en Colombia. Revista Gerencia y Políticas de Salud, vol. 9, número 19, p. 92. Recuperado de:

https://www.redalyc.org/pdf/545/54519045007.pdf

Dane. (2019). Recuperado de: https://www.dane.gov.co/files/faqs/faq_ech.pdf

Enciclopedia virtual. Recuperado de: http://www.eumed.net/tesisdoctorales/2012/mirm/enfoque_cualitativo.html 
Enciclopedia virtual. Recuperado de: http://www.eumed.net/tesis-

doctorales/2012/zll/metodologia-investigacion.html

ElColombiano. Recuperado de:

https://www.elcolombiano.com/opinion/editoriales/mujeres-y-mercado-laboral-

$\underline{\mathrm{CG} 10341517}$

Revista Dinero, (2019). Colombia: crece la brecha laboral contra la mujer. ¿Por qué?.

Recuperado de: https://www.dinero.com/management/articulo/cifras-de-la-brecha-

laboral-contra-la-mujer-en-colombia/267712

Herrera (2019). RCN Radio. Recuperado de: https://www.rcnradio.com/economia/que-

$\underline{\text { factores-estan-detras-del-alto-desempleo-de-las-mujeres-en-colombia }}$

Portafolio, (2013). Las dinàmicas del mercado laboral en Colombia. Recuperado de https://m.portafolio.co/economia/finanzas/ARTICULO-MOVILES-AMP-75368.html

El Tiempo, (2019). ¿Se ha logrado reducir la brecha laboral entre y mujeres? Recuperado de https://www.eltiempo.com/economia/sectores/brecha-laboral-entre-hombres-ymujeres-en-colombia-y-el-mundo-336164

Dinero, (2019). Ser mujer sigue siendo una desventaja en el mercado laboral colombiano. Recuperado de https://www.dinero.com/pais/articulo/cuales-son-las-cifras-de-desempleode-las-mujeres-en-colombia/275660

Redmas, (2019). En Colombia, las mujeres les es más difícil conseguir trabajo.

Recuperado de: http://www.redmas.com.co/colombia/colombia-mas-dificil-conseguir$\underline{\text { trabajo-las-mujeres/ }}$

Dinero, (2019). Desempleo: el principal problema económico de Colombia. Recuperado de: https://www.dinero.com/pais/articulo/que-pasa-con-el-desempleo-en$\underline{\text { colombia/273148 }}$ 
El Tiempo, (2019). ¿Por qué es difícil para algunos conseguir empleo es tan difícil? Recuperado de https://www.eltiempo.com/economia/sectores/por-que-es-tan-dificilconseguir-trabajo-en-colombia-137268 